\title{
Acknowledgements to Referees in 2014
}

\author{
Fawu Wang
}

\begin{abstract}
Launched in 2014, Geoenvironmental Disasters was already highly successful in its first year. Besides the appreciation to the authors who contributed their works to the journal, we want to express our sincere acknowledgement to the referees, who supported the journal with their expertise during peer review. Their qualified and constructive contribution to the journal is respected and appreciated.
\end{abstract}

\section{Editorial}

The following experts completed the peer-reviewing process of Geoenvironmental Disasters for the first volume in 2014.

Rifaat Abdalla, King Abdulaziz University, Kingdom of Saudi Arabia

Beena Ajmera, California State University, Fullerton, California, USA

Camilo Allyson, Universidade Federal de Campina Grande (UFCG), Brazil

Netra Prakash Bhandary, Ehime University, Matsuyama, Japan

Eddie Bromhead, Kingston University London, Great Britain

Rong-Her Chen, National Taiwan University, Chinese Taipei

Ranjan Kumar Dahal, Tribhuvan University, Kathmandu, Nepal

Weili Duan, Kyoto University, Japan

Adam Emmer, Charles University in Prague, Czech Republic

Joseph Gartner, United States Geological Survey, Rocky Mountain Area, USA

Giovanni Gigli, University of Firenze, Florence, Italy

Hans-Balder Havenith, Université de Liège, Belgium

Tabatabaei S. H. Heisebei, Road, Building \&Urban

Development Research Center, Iran

Lynn Highland, United States Geological Survey, USA

Ogbonnaya Igwe, University of Nigeria, Nigeria

CD Jan, National Cheng Kung University, Chinese Taipei
Ismail Ragip Karas, Karabuk University, Karabuk, Turkey

Fevzi Karsli, Karadeniz Technical University, Trabzon, Turkey

Karel Kirchner, Masaryk University, Brünn, Tschechien

Tetsuya Kogure, Shimane University, Matsue, Japan

José Cleidimário Arauújo Leite, Universitdade Federal de Campina Grande, Brazil

Tonglu Li, Chang'an University, Xi'an, China

Zhihui Z Li, Harvard University, Cambridge, USA

Enlong Liu, Sichuan University, Chengdu, China

Irene Manzella, Université de Genève, Switzerland

Masakatsu Miyajima, Kanazawa University, Japan

Ngadish Ngasisih, Gadjah Mada University, Yogyakarta, Indonesia

Mario Parise, Institute of Research for the Hydrological Protection, Bari, Italy

Veronica Pazzi, University of Florence, Italy

Bam H.N. Razfindrabe, University of the Ryukyus, Okinawa, Japan

Jean Roger, ENS, Paris, France

Jun Shen, China Institute of Disaster Prevention, Beijing, China

Yanjun Shen, Center for Agricultural Resources Research, Chinese Academy of Sciences, China

Jusong Shi, China Geological Survey, Beijing, China

Mauro S Soldati, Università di Modena e Reggio Emilia, Italy

Alexander Strom, Geodynamics Research Center, Moscow, Russia

Fubao Sun, Australian National University, Australia

Yih-Chin Tai, National Cheng Kung University, Chinese Taipei

Thanh Ngo-Duc, Hanoi University of Science, Vietnam

Ling Hong Tian, RWTH Aachen University, Germany 
Manita Timilsina, Himalaya Conservation Group, Nepal Gangalal Tuladhar, Himalaya Conservation Group, Nepal

Vit Vilímek,Charles University in Prague, Czech Republic

Guoqing Wang, Nanjing Hydraulic Research Institute, China

Huabin Wang, Huazhong University of Science and Technology, Wuhan, China

Lei Wang, Clemson University, USA

Shaun Williams, University of Canterbury, Christchurch, New Zealand

Ying-Hsin Wu, Shimane University, Matsue, Japan

Chong Xu, Institute of Geology, China Earthquake Administration, China

Jianhua Xu, East China Normal University, Shanghai, China

Masaho Yoshida, Fukui National College of Technology, Japan

Endi Zhai, Kleinfelder West, Inc, USA

Received: 21 June 2015 Accepted: 21 June 2015

Published online: 01 July 2015

Submit your manuscript to a SpringerOpen ${ }^{\circ}$ journal and benefit from:

- Convenient online submission

- Rigorous peer review

- Immediate publication on acceptance

- Open access: articles freely available online

- High visibility within the field

- Retaining the copyright to your article 